\title{
Síntomas negativos debidos a síndrome de apnea del sueño en un paciente con un trastorno delirante
}

\author{
R. Bottlender y H.-J. Möller \\ Departamento de Psiquiatría. Universidad Ludwig Maximilians, Munich, Alemania
}

En lo que sigue, comunicamos el caso de un paciente con trastorno delirante (tipo celotípico, DSM-IV: 297.1), que presentaba síntomas negativos que se debían en parte a un síndrome de apnea obstructiva del sueño (OSAS).

El paciente de 60 años fue admitido en nuestro hospital para el tratamiento de síntomas negativos que no respondían a la medicación en el contexto de un trastorno delirante. El primer comienzo de la enfermedad se había producido hacía un año. Se descartó una historia anterior de trastornos psiquiátricos o trastornos orgánicos graves. En el momento de la admisión, el paciente recibía pretratamiento psicofarmacológico con dosis altas de neurolépticos (haloperidol, $20 \mathrm{mg} \mathrm{d}-1$; decanoato de haloperidol [ $3 \mathrm{~mL}$, i.m., intervalo de 14 días], clorprotixeno, $80 \mathrm{mg} \mathrm{d}$ 1 , teraleno 10 gotas d-1). El examen neurológico reveló un síndrome parkinsonoide significativo. El estado psicopatológico estaba dominado por los síntomas afectivos, en cuanto a embotamiento afectivo, rigidez afectiva, pensamiento retardado, inhibido y restringido, falta de impulso y retirada social. El pensamiento delirante estaba sistematizado, pero con una dinámica baja. No se pudieron detectar otros síntomas psicóticos. De conformidad con la hipótesis de que los síntomas negativos, combinados con síntomas depresivos posiblemente subyacentes, eran secundarios a las altas dosis de neurolépticos, iniciamos consecutivamente las estrategias de tratamiento siguientes: reducción del haloperidol a una dosis de $1 \mathrm{mg} \mathrm{d}-1$ e interrupción de los otros pretratamientos; combinación de paroxetina (hasta $40 \mathrm{mg} \mathrm{d}-1$ ) y haloperidol (1 $\mathrm{mg} \mathrm{d}-1)$, e interrupción de la medicación anterior y comienzo de risperidona hasta $6 \mathrm{mg} \mathrm{d}-1$ como monoterapia en la novena semana de la estancia hospitalaria. Bajo el tratamiento con risperidona, se vio por primera vez, aparte de la mejoría del parkinsonismo, una mejoría en el estado psicopatológico. Sin embargo, persistían todavía en un nivel significativo síntomas negativos nucleares, en cuanto a falta de impulso, pérdida general de energía y embotamiento afectivo. En esta situación, observamos que el paciente sufría de episodios de apnea durante la noche. Se realizó una polisomnografía, que confirmó el diagnóstico de OSAS. Para tratar el OSAS, iniciamos una terapia de presión aérea positiva continua, que se asoció con una mejoría adicional de los síntomas negativos.

¿Qué importancia clínica tiene el caso comunicado? El OSAS no es una condición clínica infrecuente. La prevalencia entre los hombres de 40 a 65 años de edad es 8,5\% [1]. Los síntomas típicos del OSAS son: cansancio diurno, disfunción cognitiva difusa y múltiples secuelas emocionales y psicosociales. Estos síntomas se pueden observar también en pacientes esquizofrénicos con síntomas negativos. Por tanto, sería de esperar que los aspectos diagnósticos diferenciales del OSAS y los síntomas negativos, especialmente en los pacientes esquizofrénicos, se hubieran debatido ya en las publicaciones. Sin embargo, los resultados de una búsqueda en el sistema Medline indican que no es éste el caso (búsqueda de "apnea del sueño o OSAS, y síntomas negativos"). Basándonos en estos antecedentes y con respecto al aumento de mortalidad en los pacientes con OSAS, asumimos que la apnea del sueño en los pacientes esquizofrénicos con síntomas negativos puede estar insuficientemente diagnosticada.

\section{BIBLIOGRAFÍA}

1 Partinen M, Telakivi T. Epidemiology of obstructive sleep apnea syndrome. Sleep 1992; 15 (6 Suppl): 1-4. 